\title{
Accuracy of Different Methods of Working Length Determination in Endodontics
}

\author{
Dr. G. Midhun Mohan1, Prof. Dr. V. Susila Anand2 \\ 1(Department of Conservative Dentistry and Endodontics, Saveetha Dental College and Hospital, Saveetha \\ University, Chennai, India) \\ 2(Department of Conservative Dentistry and Endodontics, Saveetha Dental College and Hospital, Saveetha \\ University, Chennai, India)
}

\begin{abstract}
Background: Accurate assessment of the working length determines the success and prognosis of endodontic treatment. There are no systematic reviews comparing the accuracy of different methods of working length determination in endodontics

Aim: To evaluate clinical studies on the accuracy of different methods used for working length determination in endodontics.

Search strategy: Search was conducted on Pub med central, Medline and Mesh data base for the related topic from 1991 to 2012. Articles were selected, if they met the following criteria: clinical trials, clinical studies, randomized controlled trials and controlled clinical trials.

Results: There is no significant difference between conventional methods and electronic apex locators in the accuracy of working length determination. But electronic apex locators and digital radiographic methods were found to be beneficial from the perspective of radiation dose reduction.

Conclusion: Electronic apex locators are not superior to radiographs in determining working length. Long term follow up studies evaluating post operative success comparing electronic apex locators and radiographic methods are needed to appreciate the best method of working length determination in endodontics.

Key Words; Conventional radiography, Digital radiography, Electronic apex locator, Endodontics, Working length.
\end{abstract}

\section{Introduction}

In endodontics, the working length is defined as the distance from a coronal reference point to the point at which canal preparation and obturation should terminate (Glossary of endodontic terms). Accurate assessment of the working length determines the success and prognosis of endodontic treatment.

One of the major problems in endodontic treatment has always been identification and maintenance of the biological length of the root canal system. Optimal healing condition with minimal contact between the obturation material and the apical tissue is achieved when root canal treatment, terminates at the cementodentinal junction [16].

However in clinical practice, the minor apical foramen, as a more consistent anatomic feature, can be regarded as being the narrowest portion of root canal system and thus, the ideal landmark for the apical end point for root canal treatment [13]. Accurate determination of working length prevents under instrumentation that could leave tissues and debris in the apical segment, or over instrumentation which could cause patient discomfort, damage periapical tissue, or potentially cause an infection or cyst development from the placement of irritating materials beyond the apex [4].

Different methods have been used for locating the position of canal terminus and measuring the working length of root canals. These include radiographic methods, electronic methods, tactile method and other adjunctive methods.

Radiographic method, traditionally the most popular and trusted way for length measurement in the field of endodontics has many advantages, like direct observation of the anatomy of root canal system, number and curvature of roots and in addition acts as an initial guide for working length estimation [12]. There are, however a number of disadvantages like radiation hazard both to the patient and dental personnel, image distortion and observer's bias in radiographic interpretation which may lead to errors [1].

Technological advances have led to the introduction of digital radiography which has many potential benefits in endodontic practice, The first commercial integrated digital imaging system was Radiovisiography involving the use of an intraoral sensor instead of conventional X-ray film. The RVG system allowed a substantial reduction in radiation dosage and duration of endodontic procedures because it effectively eliminated the film processing time. In the same way, the zoom function had the potential to improve the diagnostic performance by magnifying areas such as the apical zone [10]. 
The development and production of electronic devices for locating the canal terminus have been major innovations in root canal treatment. An electronic method for root length determination was first conceived by Custer (1918) and the idea was revisited by Suzuki (1942); but it was Sunada, who in 1962, took these principles and constructed a simple device that used direct current to measure the canal length. Subsequently the electronic apex locators have been greatly improvised to increase their accuracy and versatility.

They are equal or higher in accuracy compared with radiographic methods and this has been shown by various, in-vivo, ex-vivo and in-vitro studies. The advantages of using electronic apex locators are that it is partially useful when apical portion of canal system is obscured by certain anatomic structures. In addition, electronic apex locators help to reduce the treatment time and radiation dose, which may be higher with conventional radiographic measurements [15].

Even though electronic apex locators are considered gold standard in endometrics, they cannot be considered a panacea for this purpose owing to their limitations. The main disadvantages of electronic apex locators are that it cannot be used in patients with cardiac pacemakers, perforations, fractures of root and their accuracy in cases of immature apex, root resorption, hemorrhage and swelling are also questionable. [6 \& 21] Thus there is no consensus on the best working length determination method in the literature. There are no systematic reviews comparing the accuracy of different methods of working length determination in endodontics.

\section{AIM}

The aim of this systematic review is to evaluate clinical studies on the different methods of working length determination in endodontics. The primary objective is to determine whether electronic apex locator is superior to radiographic method in determination of working length. The secondary objective is to compare the accuracy of various methods of working length determination.

\section{Materials And Methods}

1. "Criteria for considering studies for the review"

For the identification of studies to be considered in the review, detailed search strategies were developed for each database searched. The MEDLINE search used combination of controlled vocabulary and free text terms.

2. "Sources used"

2.1 "Searched databases"

Pubmed (from 1991 to present)

Pubmed advanced search (from 1991 to present)

Mesh

Medline

3. "Language"

Original articles in English and articles available in English translation were selected for this review.

4. "Hand search"

All issues of the following journals were hand searched as being of particular importance to the review.

Journal of Endodontics

International Endodontic Journal

Journal of American Dental Association

Journal of Dentistry

Oral Medicine, Oral Pathology, Oral Surgery, Oral Radiology and Endodontics.

British Dental Journal

Endodontic Topics

5. "Data collection and analysis"

The primary outcome was to evaluate whether electronic apex locator is superior to radiographic method in determination of working length in endodontics.

The secondary outcome included most accurate method of working length determination in endodontics.

INCLUSION CRITERIA

1. "Criteria for considering studies for this review" 
1.1 "Types of Studies" : Randomized controlled trials, clinical studies (in-vivo\&ex-vivo) evaluating various working length determination methods in endodontics in permanent dentition and which compared the accuracy of various methods of working length determination used in endodontics.

1.2 "Teeth included" : All permanent teeth.

1.3 "Types of Interventions" : Various methods used for the determination of working length in endodontics. These methods include

Conventional radiographic method using

Ingle's method

Grossman's formula

Digital radiographic method $-\mathrm{RVG}$

Electronic method i.e., using electronic apex locator (all generations) and other adjunctive methods (tactile, paper point).

\section{2. "Study selection"}

The title, keywords and abstracts of reports identified fromelectronic searching for evidence of following criteria were examined:

Clinical studies (in-vivo, ex-vivo)

Clinical trials

Randomized controlled trials

Controlled clinical trials

Working length determination

Conventional radiographic method using Ingle's method, Grossman's formula

Digital radiographic method $-\mathrm{RVG}$

Electronic methods - Electronic apex locators (all generations)

\section{EXCLUSION CRITERIA}

The following studies were excluded:

Primary teeth

In-vitro studies

Animal studies

Completely off topic or different methodology

Reviews

\section{Chart 1: SEARCH FLOW CHART}

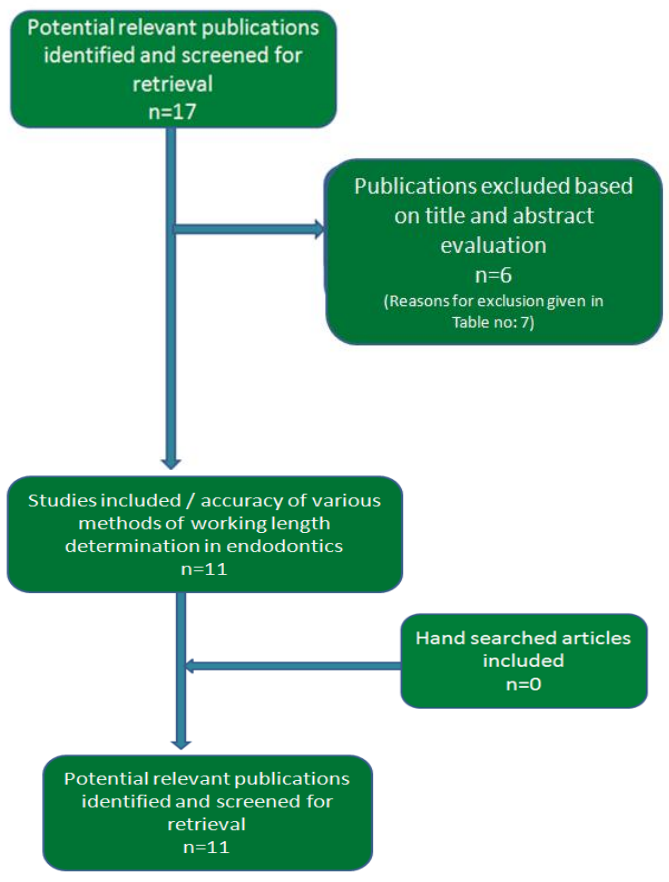


TABLE 1: VARIABLES OF INTEREST

\begin{tabular}{|l|l|}
\hline & \\
\hline $\mathbf{2}$ & Master cone fit (radiographic evaluation) \\
\hline $\mathbf{3}$ & Obturation adequacy \\
\hline $\mathbf{4}$ & Post operative pain and other symptoms \\
\hline $\mathbf{5}$ & Success of endodontic treatment \\
\hline
\end{tabular}

TABLE 2: GENERAL INFORMATION OF SELECTED ARTICLES- METHODOLOGY

\begin{tabular}{|c|c|c|c|c|c|c|}
\hline $\begin{array}{l}\text { S. } \\
\text { No. }\end{array}$ & Reference & $\begin{array}{l}\text { Study } \\
\text { Descript- } \\
\text { ion }\end{array}$ & Sample Type & Sample Size & $\begin{array}{l}\text { Controls } \\
\text { Employed }\end{array}$ & Test Group \\
\hline 1 & $\begin{array}{l}\text { Jarad F.D. et. } \\
\text { al. }\end{array}$ & RCT & $\begin{array}{l}\text { Adult teeth } \\
\text { requiring } \mathrm{RCT}\end{array}$ & $\begin{array}{l}50 \\
\text { patients }\end{array}$ & $\begin{array}{l}\text { Conventional } \\
\text { radiograph }\end{array}$ & $\begin{array}{l}\text { Electronic apex } \\
\text { Locator(Raypex 5-4th } \\
\text { generation) }\end{array}$ \\
\hline 2 & $\begin{array}{l}\text { Parekh V. et. } \\
\text { al. }\end{array}$ & Ex-vivo & $\begin{array}{l}\text { Pre-molars } \\
\text { (single root, } \\
\text { straight root) }\end{array}$ & $\begin{array}{l}20 \text { patients }-20 \\
\text { canals }\end{array}$ & No control & $\begin{array}{l}\text { Electronic apex locator (Root } \\
\text { ZX, 3rd generation) \& } \\
\text { conventional radiograph }\end{array}$ \\
\hline 3 & $\begin{array}{l}\text { Stober } \\
\text { et. al. }\end{array}$ & $\begin{array}{l}\text { In-vivo } \\
\text { study }\end{array}$ & $\begin{array}{l}\text { Premolars, } \\
\text { Canine, } \\
\text { Incisors }\end{array}$ & $\begin{array}{l}37 \text { teeth }- \\
40 \text { canals }\end{array}$ & No control & $\begin{array}{l}2 \text { electronic apex locators with } \\
\text { the help of manual } \mathrm{K} \text { files }\end{array}$ \\
\hline 4 & $\begin{array}{l}\text { Stober } \\
\text { et. al. }\end{array}$ & $\begin{array}{l}\text { In-vivo } \\
\text { study }\end{array}$ & $\begin{array}{l}\text { Premolars, } \\
\text { Canine, } \\
\text { Incisors }\end{array}$ & $\begin{array}{l}35 \text { teeth }- \\
40 \text { canals }\end{array}$ & No control group & $\begin{array}{l}2 \text { electronic apex locators }- \text { Root } \\
\text { ZX:3rd generation and iPex-4th } \\
\text { generation }\end{array}$ \\
\hline 5 & $\begin{array}{l}\text { Ravanshad } \\
\text { et. al. }\end{array}$ & RCT & $\begin{array}{l}\text { All teeth with } \\
\text { mature apices }\end{array}$ & $\begin{array}{l}84 \text { patients }- \\
188 \text { canals }\end{array}$ & No control group & $\begin{array}{l}1 \text { group-electronic apex locator } \\
\text { and 2nd group-conventional } \\
\text { radiograph }\end{array}$ \\
\hline 6 & $\begin{array}{l}\text { Smadi L. } \\
\text { et. al. }\end{array}$ & $\begin{array}{l}\text { Clinical } \\
\text { study }\end{array}$ & $\begin{array}{l}\text { All teeth } \\
\text { requiring } \mathrm{ACT}\end{array}$ & $\begin{array}{l}66 \text { patients }- \\
151 \text { canals }\end{array}$ & No control group & $\begin{array}{l}1 \text { group-electronic apex locator } \\
\text { alone and 2nd group-electronic } \\
\text { apex locator }+ \text { conventional } \\
\text { radiograph }\end{array}$ \\
\hline 7 & $\begin{array}{l}\text { Hoer D. } \\
\text { et. al. }\end{array}$ & $\begin{array}{l}\text { In-vivo } \\
\text { study }\end{array}$ & $\begin{array}{l}\text { All teeth with } \\
\text { mature apices }\end{array}$ & $\begin{array}{l}42 \text { patients - } \\
79 \text { teeth } \\
93 \text { canals }\end{array}$ & $\begin{array}{l}\text { Histological } \\
\text { section }\end{array}$ & $\begin{array}{l}2 \text { electronic apex locators - } \\
\text { Justy II and Endy } 5000\end{array}$ \\
\hline 8 & $\begin{array}{l}\text { Welk A.R. } \\
\text { et. al. }\end{array}$ & $\begin{array}{l}\text { In-vivo } \\
\text { study }\end{array}$ & $\begin{array}{l}\text { Incisor, canine, } \\
\text { premolar }\end{array}$ & 32 teeth & $\begin{array}{l}\text { Histological } \\
\text { section }\end{array}$ & $\begin{array}{l}2 \text { electronic apex locators - Root } \\
\text { ZX: } 2 \text { frequency and AFA 2005: } \\
5 \text { frequency }\end{array}$ \\
\hline 9 & $\begin{array}{l}\text { Ashraf F.F. et. } \\
\text { al. }\end{array}$ & $\begin{array}{l}\text { In-vivo } \\
\text { study }\end{array}$ & $\begin{array}{l}\text { Adult teeth } \\
\text { requiring } \mathrm{RCT}\end{array}$ & $\begin{array}{l}36 \text { teeth } \\
58 \text { canals }\end{array}$ & No control group & $\begin{array}{l}\text { Electronic apex locator and } \\
\text { conventional radiograph }\end{array}$ \\
\hline 10 & $\begin{array}{l}\text { Saad A.Y. } \\
\text { et. al. }\end{array}$ & $\begin{array}{l}\text { In-vivo } \\
\text { Study }\end{array}$ & $\begin{array}{l}\text { 6-ant.teeth } \\
\text { 4-PM } \\
\text { 4-molars }\end{array}$ & 14 teeth & $\begin{array}{l}\text { No control } \\
\text { group(master } \\
\text { cone taken with } \\
\text { RVG) }\end{array}$ & $\begin{array}{l}\text { Electronic apex locator and } \\
\text { RVG }\end{array}$ \\
\hline 11 & $\begin{array}{l}\text { Keller M.E. } \\
\text { et. al. }\end{array}$ & $\begin{array}{l}\text { In-vivo } \\
\text { Study }\end{array}$ & $\begin{array}{l}\text { 30-ant. teeth } \\
\text { 39-post. teeth }\end{array}$ & $\begin{array}{l}69 \text { teeth - } \\
99 \text { canals }\end{array}$ & $\begin{array}{l}\text { Radiograph taken } \\
\text { by experienced } \\
\text { endodontist }\end{array}$ & $\begin{array}{l}\text { Electronic apex locator } \\
\text { Endocater }\end{array}$ \\
\hline
\end{tabular}

\section{Results}

TABLE 3: GENERAL INFORMATION OF SELECTED ARTICLES

\begin{tabular}{|c|c|c|c|c|c|c|c|c|}
\hline \multirow{2}{*}{$\begin{array}{l}\text { S. } \\
\text { No. }\end{array}$} & \multirow[t]{2}{*}{ Reference } & \multirow{2}{*}{$\begin{array}{l}\text { Test } \\
\text { Group }\end{array}$} & \multicolumn{5}{|c|}{ VARIABLES OF INTEREST } & \multirow[t]{2}{*}{ Results } \\
\hline & & & $\begin{array}{l}\text { Assessment of } \\
\text { accuracy of } \\
\text { WL } \\
\text { determination }\end{array}$ & $\begin{array}{l}\text { Master } \\
\text { cone fit }\end{array}$ & $\begin{array}{l}\text { Obtura-tion } \\
\text { adequacy }\end{array}$ & $\begin{array}{l}\text { Post } \\
\text { operative } \\
\text { pain \& other } \\
\text { symptoms }\end{array}$ & $\begin{array}{l}\text { Success of } \\
\text { endodontic } \\
\text { treatment }\end{array}$ & \\
\hline 1 & $\begin{array}{l}\text { Jarad F.D. } \\
\text { et. al. }\end{array}$ & EAL & & & & & & 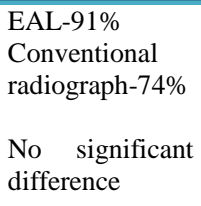 \\
\hline 2 & $\begin{array}{l}\text { Parekh V. et. } \\
\text { al. }\end{array}$ & $\begin{array}{l}\text { EAL } \\
\& \\
\text { Conven- } \\
\text { tionalrad } \\
\text { iogr-aph }\end{array}$ & & & & & & $\begin{array}{l}\text { EAL }-0.4240 \pm \\
0.4587 \\
\text { Conventional } \\
\text { radiograph }- \\
0.5430 \pm \\
0.5741\end{array}$ \\
\hline
\end{tabular}




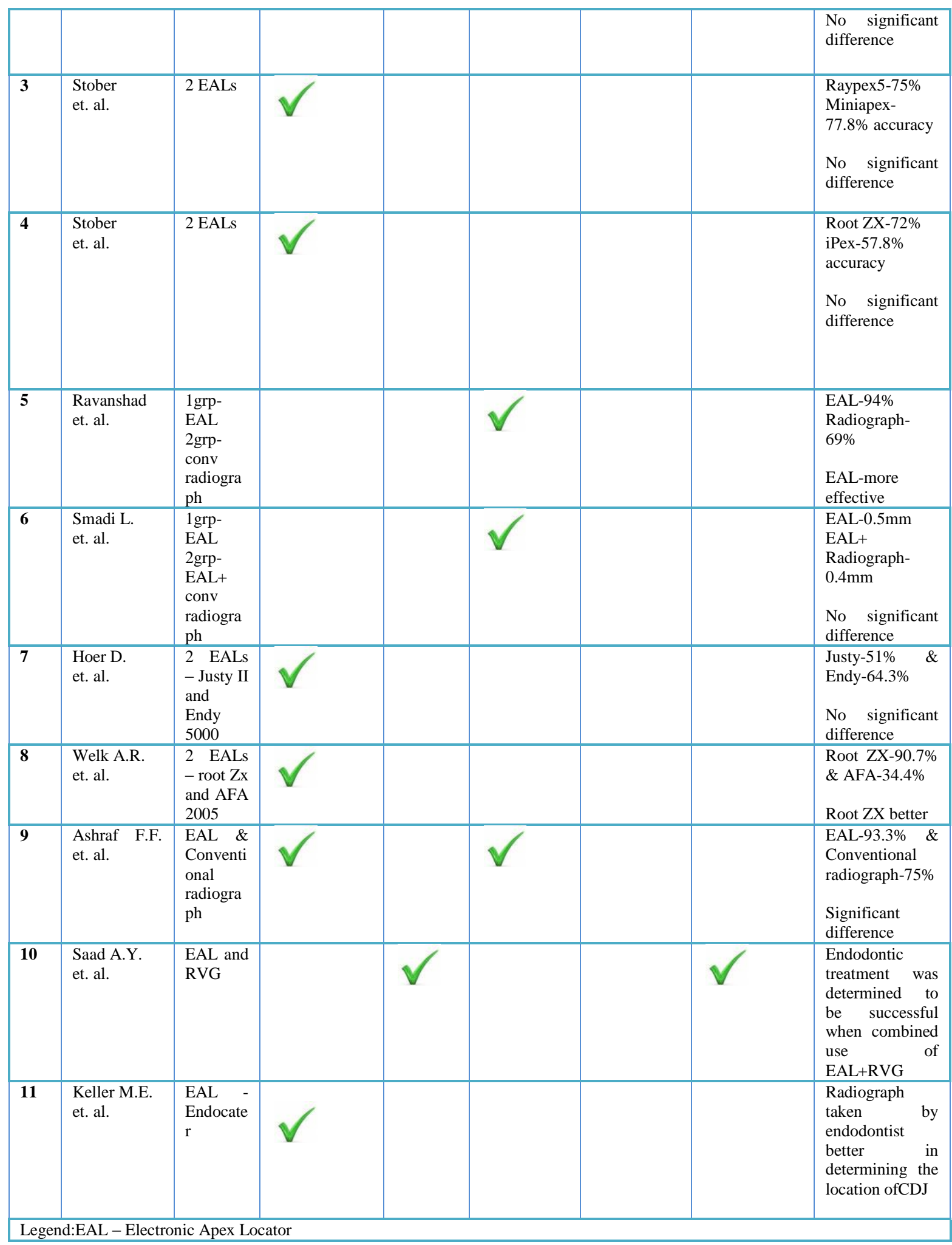


TABLE 4: EVIDENCE LEVEL OF SELECTED ARTICLES

\begin{tabular}{|c|c|c|c|c|c|c|c|c|}
\hline $\begin{array}{l}\text { S. } \\
\text { No }\end{array}$ & Reference & $\begin{array}{l}\text { Randomiz- } \\
\text { ation }\end{array}$ & $\begin{array}{l}\text { Allocation } \\
\text { concealment }\end{array}$ & $\begin{array}{l}\text { Blind- } \\
\text { ing }\end{array}$ & $\begin{array}{l}\text { Clear } \\
\text { IC/EC }\end{array}$ & $\begin{array}{l}\text { Complete- } \\
\text { ness of follow } \\
\text { up }\end{array}$ & $\begin{array}{l}\text { Sample size } \\
\text { calculation }\end{array}$ & $\begin{array}{l}\text { CEBM } \\
\text { Evidence }\end{array}$ \\
\hline 1 & $\begin{array}{l}\text { Ashraf F.F. } \\
\text { et. al. }\end{array}$ & Yes & Yes & Yes & $\begin{array}{l}\text { No } \\
\text { (only IC } \\
\text { men-tion) }\end{array}$ & NA & $\begin{array}{l}\text { Not } \\
\text { Mentioned }\end{array}$ & 2 \\
\hline 2 & $\begin{array}{l}\text { Parekh V. et. } \\
\text { al. }\end{array}$ & Yes & $\begin{array}{l}\text { Not } \\
\text { Mentioned }\end{array}$ & No & Yes & NA & $\begin{array}{l}\text { Not } \\
\text { Mentioned }\end{array}$ & 3 \\
\hline 3 & $\begin{array}{l}\text { Stober } \\
\text { et. al. }\end{array}$ & Yes & $\begin{array}{l}\text { Not } \\
\text { Mentioned }\end{array}$ & Yes & No & NA & $\begin{array}{l}\text { Not } \\
\text { Mentioned }\end{array}$ & 3 \\
\hline 4 & $\begin{array}{l}\text { Stober } \\
\text { et. al. }\end{array}$ & Yes & $\begin{array}{l}\text { Not } \\
\text { Mentioned }\end{array}$ & Yes & No & NA & $\begin{array}{l}\text { Not } \\
\text { Mentioned }\end{array}$ & 3 \\
\hline 5 & $\begin{array}{l}\text { Ravanshad } \\
\text { et. al. }\end{array}$ & Yes & $\begin{array}{l}\text { Not } \\
\text { Mentioned }\end{array}$ & Yes & Yes & NA & $\begin{array}{l}\text { Not } \\
\text { Mentioned }\end{array}$ & 2 \\
\hline 6 & $\begin{array}{l}\text { Smadi L. } \\
\text { et. al. }\end{array}$ & Yes & $\begin{array}{l}\text { Not } \\
\text { Mentioned }\end{array}$ & $\begin{array}{l}\text { Not } \\
\text { Mention } \\
\text { ed }\end{array}$ & No & NA & $\begin{array}{l}\text { Not } \\
\text { Mentioned }\end{array}$ & 3 \\
\hline 7 & $\begin{array}{l}\text { Hoer D. } \\
\text { et. al. }\end{array}$ & Yes & $\begin{array}{l}\text { Not } \\
\text { Mentioned }\end{array}$ & $\begin{array}{l}\text { Not } \\
\text { Mention } \\
\text { ed }\end{array}$ & No & NA & $\begin{array}{l}\text { Not } \\
\text { Mentioned }\end{array}$ & 3 \\
\hline 8 & $\begin{array}{l}\text { Welk A.R. } \\
\text { et. al. }\end{array}$ & No & $\begin{array}{l}\text { Not } \\
\text { Mentioned }\end{array}$ & Yes & No & NA & $\begin{array}{l}\text { Not } \\
\text { Mentioned }\end{array}$ & 3 \\
\hline 9 & $\begin{array}{l}\text { Ashraf F.F. } \\
\text { et. al. }\end{array}$ & Yes & $\begin{array}{l}\text { Not } \\
\text { Mentioned }\end{array}$ & Yes & No & NA & $\begin{array}{l}\text { Not } \\
\text { Mentioned }\end{array}$ & 3 \\
\hline 10 & $\begin{array}{l}\text { Saad A.Y. } \\
\text { et. al. }\end{array}$ & No & $\begin{array}{l}\text { Not } \\
\text { Mentioned }\end{array}$ & $\begin{array}{l}\text { Not } \\
\text { Mention } \\
\text { ed }\end{array}$ & No & Yes & $\begin{array}{l}\text { Not } \\
\text { mentioned }\end{array}$ & 3 \\
\hline 11 & $\begin{array}{l}\text { Keller M.E. } \\
\text { et. al. }\end{array}$ & No & $\begin{array}{l}\text { Not } \\
\text { Mentioned }\end{array}$ & $\begin{array}{l}\text { Not } \\
\text { Mention } \\
\text { ed }\end{array}$ & No & NA & $\begin{array}{l}\text { Not } \\
\text { mentioned }\end{array}$ & 3 \\
\hline
\end{tabular}

TABLE 5: RISK OF BIAS OF SELECTED ARTICLES MAJOR CRITERIA

\begin{tabular}{|c|c|c|c|c|c|c|}
\hline S. No. & Reference & $\begin{array}{l}\text { Method of } \\
\text { Randomization }\end{array}$ & $\begin{array}{l}\text { Allocation } \\
\text { Concealment }\end{array}$ & Blinding & Dropout Rate & Risk of Bias \\
\hline 1 & Jarad F.D. et. al. & $\begin{array}{l}\text { Not } \\
\text { Mentioned }\end{array}$ & & & NA & Low \\
\hline 3 & $\begin{array}{l}\text { Stober } \\
\text { et. al. }\end{array}$ & $\begin{array}{l}\text { Not } \\
\text { Mentioned }\end{array}$ & $\begin{array}{l}\text { Not } \\
\text { Mentioned }\end{array}$ & & NA & Moderate \\
\hline 5 & $\begin{array}{l}\text { Ravanshad } \\
\text { et. al. }\end{array}$ & $\begin{array}{l}\text { Flipping } \\
\text { Coin }\end{array}$ & $\begin{array}{l}\text { Not } \\
\text { Mentioned }\end{array}$ & & NA & Low \\
\hline 6 & $\begin{array}{l}\text { Smadi L. } \\
\text { et. al. }\end{array}$ & $\begin{array}{l}\text { Not } \\
\text { Mentioned }\end{array}$ & $\begin{array}{l}\text { Not } \\
\text { Mentioned }\end{array}$ & $\begin{array}{l}\text { Not } \\
\text { Mentioned }\end{array}$ & NA & High \\
\hline
\end{tabular}


Accuracy Of Different Methods Of Working Length Determination In Endodontics

\begin{tabular}{|l|l|l|l|l|l|l|}
\hline $\mathbf{9}$ & Ashraf F.F. et. al. & $\begin{array}{l}\text { Not } \\
\text { Mentioned }\end{array}$ & $\begin{array}{l}\text { Not } \\
\text { Mentioned }\end{array}$ & NA & Moderate \\
\hline $\mathbf{1 0}$ & $\begin{array}{l}\text { Saad A.Y. } \\
\text { et. al. }\end{array}$ & $\begin{array}{l}\text { Not } \\
\text { Mentioned }\end{array}$ & $\begin{array}{l}\text { Not } \\
\text { Mentioned }\end{array}$ & $\begin{array}{l}\text { Not } \\
\text { Mentioned }\end{array}$ & $\begin{array}{l}\text { Not } \\
\text { Mentioned }\end{array}$ & High \\
\hline $\mathbf{1 1}$ & $\begin{array}{l}\text { Keller M.E. } \\
\text { et. al. }\end{array}$ & $\begin{array}{l}\text { Not } \\
\text { Mentioned }\end{array}$ & $\begin{array}{l}\text { Not } \\
\text { Mentioned }\end{array}$ & Not Mentioned & NA & High \\
\hline
\end{tabular}

TABLE 6: RISK OF BIAS OF SELECTED ARTICLES MINOR CRITERIA

\begin{tabular}{|c|c|c|c|c|c|}
\hline S. No. & Reference & Sample justified & Baseline comparison & $\begin{array}{l}\mathrm{IC} / \\
\mathrm{EC}\end{array}$ & $\begin{array}{l}\text { Method } \\
\text { Error }\end{array}$ \\
\hline 1 & $\begin{array}{l}\text { Jarad F. D. } \\
\text { et. al. }\end{array}$ & No & NA & No & No \\
\hline 2 & $\begin{array}{l}\text { Parekh V. } \\
\text { et. al. }\end{array}$ & No & NA & Yes & No \\
\hline 3 & $\begin{array}{l}\text { Stober } \\
\text { et. al. }\end{array}$ & No & NA & No & No \\
\hline 4 & $\begin{array}{l}\text { Stober } \\
\text { et. al. }\end{array}$ & No & NA & No & No \\
\hline 5 & $\begin{array}{l}\text { Raavanshad } \\
\text { et. al. }\end{array}$ & No & NA & Yes & No \\
\hline 6 & $\begin{array}{l}\text { Smadi L. } \\
\text { et. al. }\end{array}$ & No & NA & No & No \\
\hline 7 & $\begin{array}{l}\text { Hoer D. } \\
\text { et. al. }\end{array}$ & No & NA & No & No \\
\hline 8 & $\begin{array}{l}\text { Welk A.R. } \\
\text { et. al. }\end{array}$ & No & NA & No & No \\
\hline 9 & $\begin{array}{l}\text { Ashraf F.F. } \\
\text { et. al. }\end{array}$ & No & NA & No & No \\
\hline 10 & $\begin{array}{l}\text { Saad A.Y. } \\
\text { et. al. }\end{array}$ & No & NA & No & No \\
\hline 11 & $\begin{array}{l}\text { Keller M.E. } \\
\text { et. al. }\end{array}$ & No & NA & No & No \\
\hline
\end{tabular}

TABLE 7: EXCLUDED ARTICLES

\begin{tabular}{|l|l|l|}
\hline $\begin{array}{l}\text { S. } \\
\text { No. }\end{array}$ & Article & Reason for exclusion \\
\hline $\mathbf{1}$ & Herrera M. et. al., 2011 & In-vitro study \\
\hline $\mathbf{2}$ & Odabas M.E. et. al., 2011 & Evaluation in primary molars \\
\hline $\mathbf{3}$ & Pascon E.A. et. al., 2009 & In-vitro study \\
\hline $\mathbf{4}$ & Topuz O. et. al., 2007 & In-vitro study \\
\hline $\mathbf{5}$ & & \\
\hline $\mathbf{6}$ & Lu Y.M. et. al., 2006 & Article in Chinese \\
\hline
\end{tabular}

TABLE 8: SUMMATION TABLE FOR INDIVIDUAL PARAMETER

8.1 Assessment of accuracy

\begin{tabular}{|c|c|c|c|c|}
\hline S. No. & Comparison & No. of studies & Effective method & $\begin{array}{l}\text { No difference } \\
\text { between groups }\end{array}$ \\
\hline 1 & 2 EALs & 1 & - & 1 \\
\hline 2 & 2 EALs & 1 & - & 1 \\
\hline 3 & 2 EALs & 1 & - & 1 \\
\hline 4 & 2 EALs & 1 & 1(Root ZX) & - \\
\hline 5 & $\begin{array}{l}\text { EAL vs Conventional } \\
\text { radiograph }\end{array}$ & 1 & $1(\mathrm{EAL})$ & - \\
\hline 6 & $\begin{array}{l}\text { EAL vs Conventional } \\
\text { radiograph }\end{array}$ & 1 & - & 1 \\
\hline 7 & $\begin{array}{l}\text { EAL vs Conventional } \\
\text { radiograph }\end{array}$ & 1 & $\begin{array}{l}1 \text { (Conventional } \\
\text { radiograph) }\end{array}$ & - \\
\hline
\end{tabular}


8.2. Master cone fit

\begin{tabular}{|l|l|l|l|l|}
$\mathbf{S}$ S. No. & Comparison & No. of studies & Effective method & $\begin{array}{l}\text { No difference } \\
\text { between groups }\end{array}$ \\
\hline $\mathbf{1}$ & $\begin{array}{l}\text { EAL vs conventional } \\
\text { radiograph }\end{array}$ & 1 & - & 1 \\
\hline $\mathbf{2}$ & EAL vs RVG & 1 & $\begin{array}{l}\text { 1-EAL(no } \\
\text { comparison/no control) }\end{array}$ & - \\
\hline
\end{tabular}

8.3. Obturation adequacy

\begin{tabular}{|l|l|l|l|l|}
\hline S. No. & Comparison & No. of studies & Effective method & $\begin{array}{l}\text { No difference between } \\
\text { groups }\end{array}$ \\
\hline $\mathbf{1}$ & $\begin{array}{l}\text { EAL vs. conventional } \\
\text { radiograph }\end{array}$ & 1 & 1 (EAL) & - \\
\hline $\mathbf{2}$ & $\begin{array}{l}\text { EAL alone } \\
\text { vs.EAL+conventional } \\
\text { radiograph }\end{array}$ & 1 & - & 1 \\
\hline $\mathbf{3}$ & $\begin{array}{l}\text { EAL vs. conventional } \\
\text { radiograph }\end{array}$ & 1 & 1 (EAL) & - \\
\hline
\end{tabular}

8.4. Post operative pain

\begin{tabular}{|l|l|l|l|l|}
\hline S. No. & Comparison & No. of studies & Effective method & $\begin{array}{l}\text { No difference } \\
\text { between groups }\end{array}$ \\
\hline $\mathbf{1}$ & EAL vs.RVG & 1 & $\begin{array}{l}\text { 1-EAL(no } \\
\text { comparison/no } \\
\text { control) }\end{array}$ & - \\
\hline
\end{tabular}

8.5. Success of endodontic treatment

\begin{tabular}{|l|l|l|l|l|}
\hline S. No. & Comparison & No. of studies & Effective method & $\begin{array}{l}\text { No difference } \\
\text { between groups }\end{array}$ \\
\hline $\mathbf{1}$ & EAL vs.RVG & 1 & $\begin{array}{l}\text { 1-EAL(no } \\
\text { comparison/no control) }\end{array}$ & - \\
\hline
\end{tabular}

TABLE 9: OVERALL COMPARISON OF DIFFERENT METHODS OF WORKING LENGTH DETERMINATION

\begin{tabular}{|l|l|l|l|}
\hline Total No. of studies & $\begin{array}{l}\text { Electronic apex locator }- \\
\text { more effective }\end{array}$ & $\begin{array}{l}\text { Other methods used - more } \\
\text { effective }\end{array}$ & No difierence \\
\hline $\mathbf{9 *}$ & 2 & 1 & 6 \\
\hline
\end{tabular}

Legend: $* 2$ studies were excluded from this table as one of them did not have a comparison/control group and the other compared 2 different EAL's

TABLE 10: SUMMATION TABLE FOR BLINDING

\begin{tabular}{|l|l|}
\hline Total no. of studies & No. of blinding done \\
\hline $\mathbf{1 1}$ & 6 \\
\hline
\end{tabular}


PIE-CHART FOR BLINDING

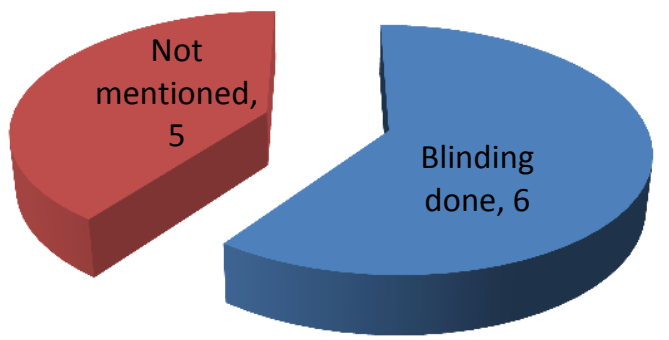

GRAPH 1: INCLUDED ARTICLES

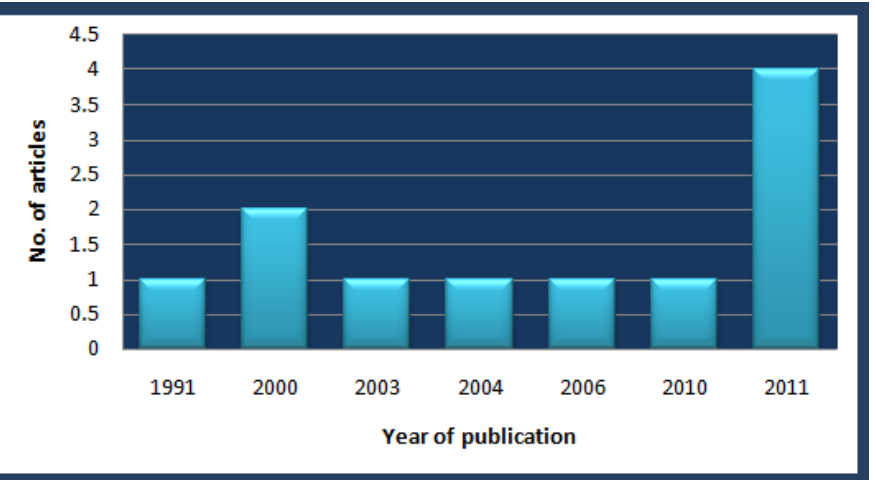

GRAPH 2: STUDY DESIGNS

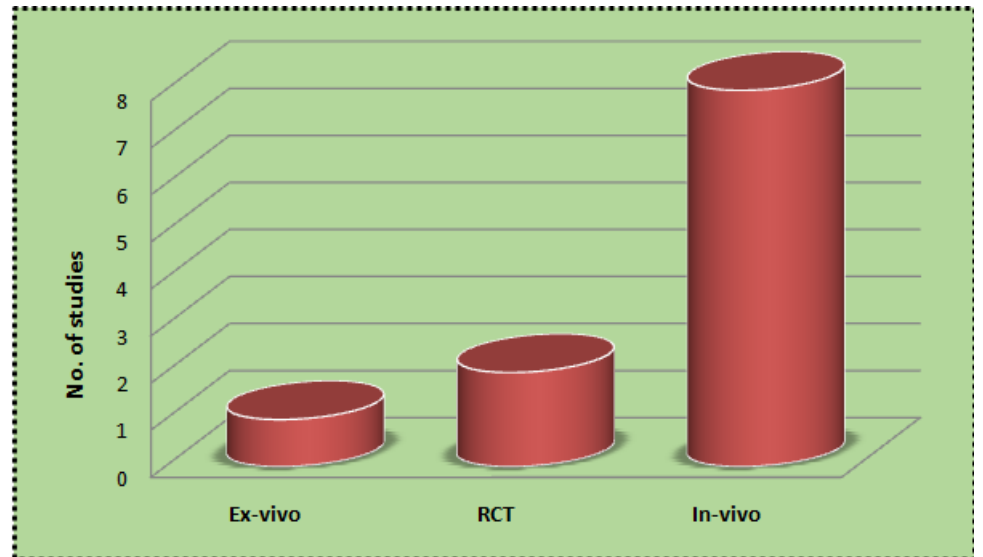

GRAPH 3: OVERALL COMPARISON OF VARIABLES OF INTEREST

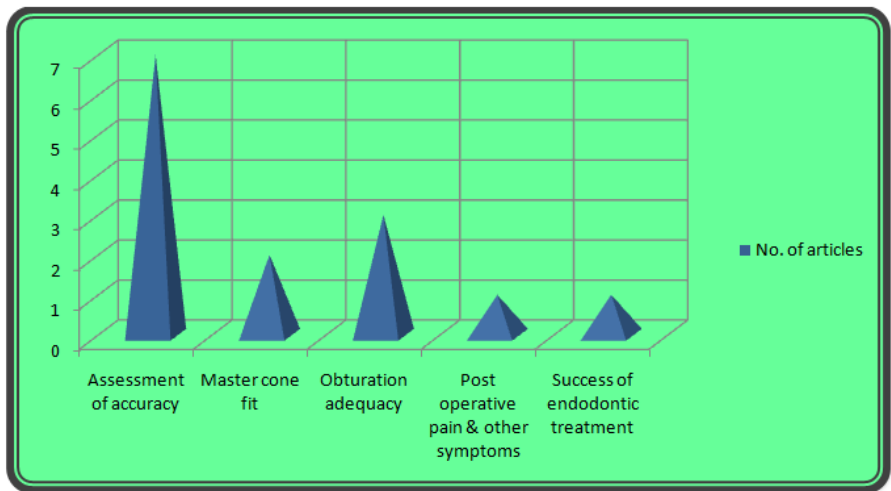


GRAPH 4: RISK OF BIAS

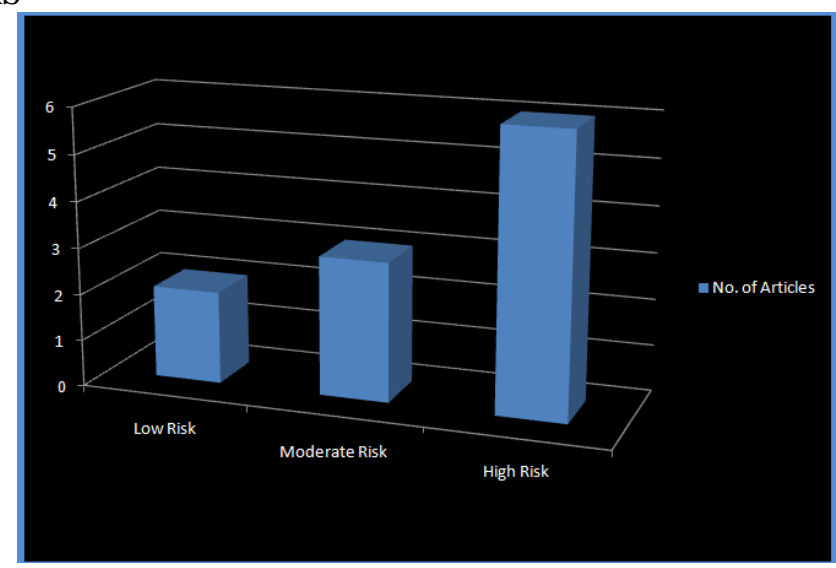

\section{Discussion}

Eleven articles are included for the systematic review. Of this, five articles aimed at evaluating the ability of electronic apex locators as an effective tool in determining working length in comparison to conventional working length radiograph in general dental practice. Another four articles compared the accuracy of two different types / generations of electronic apex locators in determining working length. One article compared the effectiveness of working length determination of an electronic apex locator used alone orin combination with a conventional working length radiograph. One article assessed the success of endodontic treatment when an electronic apex locator is used.

Out of the five articles which evaluated the ability of electronic apex locator in accurately determining working length in comparison to conventional radiographs, two articles were randomized controlled clinical trials being performed under truly clinical conditions and these aimed at providing high level of evidence for clinicians.

The Five variables of interest looked upon in this systematic revieware:

Assessment of accuracy of working length determination

Master cone fit

Obturation adequacy

Post operative pain \& other symptoms

Success of endodontic treatment

\section{1. "Interpretation of results"}

The endodontic literature puts forth randomized controlled clinical trials in-vivo \& ex-vivo studies to determine the accuracy of different methods of working length determination.

A randomized controlled clinical trial published by F.D. Jaradet. et al, (2011) evaluates the ability of apex locators as a tool in determining working length in comparison to traditional working length radiographs in general dental practice. Electronic apex locator was employed as the test group and conventional radiograph was employed as the control.

In this study, the electronic apex locator used for working length determination was Raypex 5, a fourth generation apex locator that uses two separate range of frequencies. The values obtained were of $91 \%$ accuracy for electronic apex locator when compared to $74 \%$ accuracy when conventional radiograph was used. The observed differences were not statistically significant at $5 \%$ level. In this study, comparison was based on the acceptability of master cone fit, evaluated by radiograph.

An ex-vivo study published by Parekh V. et. al. (2011) compared the accuracy of working length estimation of electronic apex locator and conventional radiograph. Root ZX was the electronic apex locator used in this study, which is a third generationapex locator. No control group was employed. Study was performed only on premolars scheduled for extraction owing to periodontal reasons having intact, single, straight root canal. After extraction, stereo-microscope was used for confirmation and comparison of both test groups. Values obtained were $0.4240 \pm 0.4587$ for apex locator when compared to $0.5430 \pm 0.5741$ for radiographs. Observed difference was not statistically significant but intra-group significance was present for both techniques. In the study, assessment of accuracy was based on the estimation of accurate location of major apical foramen.

An in-vivo study published by Stoberet. et al. (2011) compared the accuracies of working length determination of two different generations of electronic apex locators. The two electronic apex locators used were Raypex 5 - a fourth generation apex locator that uses two range of frequencies and Mini apex locator - a third generation unit that uses digital signal processing. The values obtained were of $75 \%$ accuracy for Raypex 5 
when compared to $77.8 \%$ accuracy for Mini apex locator. The observed differences were not statistically significant. In this study, the assessment of accuracy was based on the ability of both apex locators to locate a position $0.5 \mathrm{~mm}$ coronal to major foramen, and this was observed by trimming apical $4 \mathrm{~mm}$ portion of each root of the tooth being tested after extracting it and observing it under an electron microscope.

An in-vivo study published by Stoberet. al. (2011) compared the accuracies of working length of two different generations of electronic apex locators. The two different electronic apex locators used were Root $\mathrm{Zx}-$ a third generation apex locator that uses the ratio method to measure the root canal length, and iPex - a fourth generation apex locator which measures capacitance and resistance simultaneously to determine location of file tip in the canal. The values obtained were of $72 \%$ accuracy for root $\mathrm{Zx}$ when compared to $57.8 \%$ accuracy for iPex, and the observed difference was not statistically significant. In this study, the assessment of accuracy was based on the ability of both apex locators to locate a position $0.5 \mathrm{~mm}$ coronal to major foramen, and this was observed by trimming apical $4 \mathrm{~mm}$ portion of each root of the tooth being tested after extracting it and observing it under an electron microscope.

A randomized clinical trial published by Ravanshadet. al. (2010), evaluates the efficiency of working length determination by radiograph or electronic apex locator on the adequacy of final working length. The electronic apex locator used was Raypex 5 - a fourth generation apex locator that uses two ranges of frequencies. The values obtained were of $94 \%$ accuracy for electronic apex locator when compared to $69 \%$ for radiograph. The observed differences were statistically significant and the authors concluded that electronic apex locator is superior to radiographic length measurement and also decreases the rate of over estimation of length. In this study, comparison was based on the adequacy of final obturation, being evaluated by radiographs.

A clinical study (in-vivo study) published by L. Smadiet, al. (2006), compared between two methods of working length determination and its effect on radiographic extent of root canal fillings. In the study, comparison was done between an electronic apex locator used alone and also in combination with a conventional radiograph. The electronic apex locator used was Tri Auto ZX, which uses EMR Mode- electronic measurement of root canal. The values obtained were according to measurement of the mean distance from tip of root canal filling to radiographic apex, using magnifying loupes and in the electronic apex locator group it was ' $0.5 \mathrm{~mm}$ ' when compared to ' $0.4 \mathrm{~mm}$ ' in the electronic apex locator plus conventional radiograph group. The observed differences were not statistically significant, and the authors concluded that the correct use of an apex locator alone could prevent that need for further diagnostic radiographs for determination of working length.

An in-vivo study published by D. Hoer et. al. (2004), determined the accuracy of two impedance quotient apex locators under clinical conditions. The apex locators used in this study were Justy II and Endy 5000. The results of clinical measurement were controlled histologically. The values obtained were of $51 \%$ accuracy for Justy II when compared to $64.3 \%$ accuracy for Endy 5000. The observed differences were not statistically significant. In this study, assessment of accuracy was based on the accurate determination of apical constriction on the tested tooth root.

An in-vivo study published by Welk A.R. et al. (2003) compared the accuracy in detecting the minor diameter by two different types of electronic apex locators. The two different electronic apex locators used were Root ZX and Endo Analyzer 8005 and both were frequency based apex locators. Root ZX is a two frequency based electronic apex locator and Endo Analyzer 8005 is a five frequency based electronic apex locator. The values obtained were of $90.7 \%$ accuracy for Root ZX when compared to $34.4 \%$ accuracy for Endo Analyzer 8005. The observed difference was statistically significant and the Root ZX was found to be highly accurate in locating the minor diameter to within $0.5 \mathrm{~mm}$ distance. In this study, assessment of accuracy was based on the determination of accurate location of minor diameter under clinical conditions.

An in-vivo study published by Ashraf F.F. et. al. (2000) evaluates the effect of using electronic apex locator on selected endodontic treatment parameters. This study was designed to determine the value of electronic apex locators in reducing the number of working radiographs in patients, to determine the effect of using electronic apex locator versus a preoperative radiograph to estimate the working length, on the adequacy of length of final endodontic obturation and also to compare the closeness of electronic and radiographic estimates of the working length with the final working length used. The apex locator used was Root ZX - a third generation electronic apex locator .The values obtained were 93.3\% accuracy for apex locator when compared to $75 \%$ accuracy for conventional radiograph. The observed difference was statistically significant and electronic apex locator was found to be better, and the authors concluded that using an electronic estimate before radiograph verification enhances length control throughout the treatment, improves the length of obturation from the apex and reduces the number of total radiographs.

An in-vivo study published by Saad A.Y. et. al (2000) evaluated a new technique for radiation dose reduction during endodontic therapy, by using the apex locator to determine the working length. This study assessed success of endodontic treatment based on the master cone fit, evaluated with the help of radiographs. The electronic apex locator used was Root ZX - a third generation electronic apex locator. The result obtained was that the determined working lengths of the canals using electronic apex locator were comparable to the 
master cone position as estimated by RVG, with no statistical difference. The treated patients were followed up for 6 to 18 months for evaluation clinically and radiographically for post operative complications. The study concluded that a successful estimation technique can be performed by a single radiographic exposure using RVG for master cone evaluation and this technique is useful in medically compromised patients.

A in-vivo study published by Keller et al. (1991) evaluated clinically an electronic apex locator, the Endocater. The assessment of accuracy of the apex locator based on determination of the location of apical constriction or cemento-dentinal junction (CDJ), evaluated with the help of microscope after adequate sectioning of tested tooth. The Endocater is an apex locator, and its circuitry is based on the electric phenomenon that under certain conditions, impedance (resistance) is greatest at the narrowest point of the canal. The result obtained was of 51.5\% accuracy for Endocater compared to $80.2 \%$ with the help of radiograph in determining the apical constriction. The observed difference was statistically significant and the conclusion was that the radiograph taken by an experienced endodontist was better in determining the location of CDJ.

\section{2. "Defending the Results"}

The included articles describe on two randomized controlled clinical trials and nine clinical studies. In both randomized controlled clinical trials, the ability of electronic apex locators in accurately determining the working length in comparison to conventional radiograph was evaluated. Both the randomized controlled trials were performed under truly clinical conditions and thus provide high level of evidence for clinicians.

The other nine studies were clinical studies which included both in-vivo and ex-vivo studies. All studies employed standardized recognized endodontic techniques and all measurements were also made comparable by ensuring that working length recording using all techniques tested were standardized.

Four studies out of the nine clinical studies compared the accuracy of two different types / generations of electronic apex locators in determining working length. The accuracy of working length determination of all generations of electronic apex locators was assessed based on the percentage values of successful determination of apical constriction of root, the reason for this being that the apical constriction could be located consistently. Precise examination of position of file tip in relation to the apical constriction was only possible if the teeth were examined histologically with the help of a microscope after extraction.

One ex-vivo study compared the accuracy of working length estimation of two techniques, electronic apex locator and periapical radiograph. The assessment of accuracy was based on effectiveness of both techniques to locate the position of apical foramen. The reason for using apical foramen as reference point was that it gives more consistency than apical constriction or radiographic apex and its use is more reproducible for accuracy studies. An intra-group significance was observed for both techniques, the reason being that since same tooth was not employed for both techniques used.

In one in-vivo study, the success of endodontic treatment was assessed by comparing the effectiveness of working length determination using apex locator alone or in combination with working length radiograph, on apical extent of root canal filling. This was a clinical study that incorporated all errors which may occur in the mouth.

One in-vivo study determined value of electronic apex locator in reducing number of radiographs, effectiveness of electronic apex locator versus a pre-operative radiograph to estimate working length and compared closeness of electronic and radiograph estimates of working length with final working length. In this study, the author chose a completely different method of reducing bias by making sure that the practitioners were unaware of how the measurements were obtained, and this approach helped in observing that use of an electronic estimate leads to a more acceptable outcome with respect to the length of obturation termination from the apex.

In another in-vivo study, post-operative complications and success of endodontic treatment were assessed by using electronic apex locator alone in determining the working length. No control group / comparative method were present. Electronic apex locator used in this study worked even in the presence of electrolytes or vital pulp tissue. Favorable results were obtained for this study with all tested teeth and this was confirmed both by post-operative radiographs (using RVG) and follow-up radiographs (conventional).

In the last included article, an in-vivo study was performed to clinically evaluate an apex locator - Endocater, in determining the location of apical constriction or cemento-dentinal junction. The result obtained helped in acceptance of clinical applicability of the tested device, while the endodontist could successfully adjust the electronically generated file length radiograph to acceptable clinical standards without even the use of any measuring device for assistance in length measurements.

3. "Report on quality of the evidence looked upon"

Randomized controlled clinical trials evaluating the accuracy of various methods of working length determination in endodontics are extremely rare, as it was found in the present review. A few of the studies taken for endodontic working length estimation had to be excluded for specific reasons like in-vitro studies, 
studies performed on primary teeth and other language articles. Articles referenced in this review are randomized controlled clinical trials and clinical studies(in-vivo and ex-vivo).Hence, categorized as levels 2 and3respectively. Regarding the risk of bias of the included articles, 2 articles had low risk, 3 articles had moderate risk and 6 of them had high risk of bias.

\section{4. "Report on Outlier Data" \\ No outlier data was obtained.}

\section{INFERENCE}

There is no significant difference between conventional methods and electronic apex locators in the accuracy of working length determination. But electronic apex locators and digital radiographic methods were found to be beneficial from the perspective of radiation dose reduction. No long term post operative follow up was done in most of the studies barring one, where there was no proper comparison group.

\section{SUMMARY}

Establishing the length of the root canal system at the apical constriction is considered an ideal working length for endodontic treatment. In order to achieve an effective biomechanical preparation and obturation of root canal system, it is necessary for this measurement to be as accurate as possible. The aim of this systematic review is to evaluate clinical studies on the different methods of working length determination.

For the studies to be considered in the review, detailed search strategies were developed for each database searched, which include Pubmed, Pubmed Advanced search and Medline. Studies were selected if they met the following criteria: clinical trials, Randomized controlled trials, In-vivo and Ex-vivo studies comparing various methods of working length determination. The search for the related topic from 1991 to present identified 17 publications, out of which 6 were excluded after reviewing title and abstract and 11 potentially relevant publications were identified and screened for retrieval. This review included 2 randomized controlled trials and 9 clinical studies (in-vivo and ex-vivo) and hence categorized as evidence levels 2 and 3 respectively.

From the results it can be interpreted that there is no significant difference between conventional methods and electronic apex locators in the accuracy of working length determination but electronic apex locators and digital radiographic methods were found to be beneficial from the perspective of radiation dose reduction. The conclusion drawn from this systematic review is that electronic apex locators are not superior to radiographs in determining working length.

\section{Conclusion}

Electronic apex locators are not superior to radiographs in determining working length. There is no statistical difference between any two methods in accuracy.

Thus more long term randomized controlled follow up studies evaluating post operative success comparing electronic apex locators and radiographic methods are needed to appreciate the best method of working length determination in endodontics.

\section{References}

[1]. Bramante C.M., Berbert A. - A critical evaluation of some methods of determining tooth length - Oral Surgery, Oral Medicine, Oral Pathology, Oral Radiology, and Endodontics, 1974; 37:463.

[2]. Chicago I.L., in Glossary of Endodontic Terms,7th Edition in Ingle's Endodontics Ed :Ingle ,Bakland , Baumgartner 6th Edition, Year 2008 BC Decker Inc. New York

[3]. Fouad A.F., Reid L.C. - Effect of using electronic apex locators on selected endodontic treatment parameters - Journal of Dental Education, 2000

[4]. Gutmann J.L., Leonard J.E. - Problem solving in endodontic working-length determination - Compendium of Continuing Education in Dentistry, 1995; 16:288

[5]. Herrera M., Abalos C., Lucena C., Jimenez-Planas A., Llamas R. -Critical diameter of apical foramen and of file size using the Root ZX apex locator: an in vitro study - Journal of Endodontics, 2011 Sep; 37(9):1306-9.

[6]. Hoer D., Attin T. - The accuracy of electronic working length determination - International Endodontic Journal, 2004 Feb; 37(2):125-31.

[7]. Jarad F.D., Albadri S., Gamble C., Burnside G., Fox K., Ashley J.R., Peers G., Preston A.J. - Working length determination in general practice: a randomized controlled trial - British Dental Journal, 2011 Dec; 211(12):595-8.

[8]. Keller M.E., Brown C.E. Jr., Newton C.W. - A clinical evaluation of the Endocater: an electronic apex locator - Journal of Endodontics, 1991 Jun; 17(6):271-4

[9]. Lu Y.M., Qin J.N., Cao D.J. - A study on the accuracy of electronic root canal length measurement and its influential factors Shanghai Kou Qiang Yi Xue, 2006 Apr; 15(2):140-2.

[10]. Nair M.K., Nair U.P. - Digital and advanced imaging in endodontics: a review - Journal of Endodontics, $2007 ; 33: 1$.

[11]. Odabas M.E., Bodur H., Tulunoglu O., Alacam A. - Accuracy of an electronic apex locator: a clinical evaluation in primary molars with and without resorption -Journal of Clinical Pediatric Dentistry, 2011 Spring; 35(3):255-8.

[12]. Olson A.K., Goerig A.C., Cavataio R.E., Luciano J. - The ability of the radiograph to determine the location of the apical foramen International Endodontic Journal, 1991; 24:28.

[13]. Palmer M.J., Weine F.S., Healey H.J. - Position of the apical foramen in relation to endodontic therapy - Journal of the Canadian Dental Association, 1971; 37:305. 
[14]. Parekh V., Taluja C. - Comparative study of periapical radiographic techniques with apex locator for endodontic working length estimation: and ex vivo study - Journal of Contemporary Dental Practice, 2011 Mar 1; 12(2):131-4.

[15]. Pascon E.A., Marrelli M., Congi O., Ciancio R., Miceli F., Versiani M.A. -An ex vivo comparison of working length determination by three electronic apex locators - Oral Surgery, Oral Medicine, Oral Pathology, Oral Radiology, and Endodontics, 2009 Sep; 108(3):147-51

[16]. Ravanshad S., Adl A., Anvar J. - Effect of working length measurement by electronic apex locator or radiography on the adequacy of final working length: a randomized clinical trial - Journal of Endodontics, 2010 Nov; 36(11):1753-6.

[17]. Saad Y., Al-Nazhan S. - Radiation dose reduction during endodontic therapy: a new technique combining an apex locator (Root ZX) and a digital imaging system (RadioVisioGraphy) - Journal of Endodontics, 2000 Mar; 26(3):144-7.

[18]. Smadi L. - Comparison between two methods of working length determination and its effect on radiographic extent of root canal filling: a clinical study - BMC Oral Health, 2006 Feb; 11;6:4.

[19]. Stober E.K., De Ribot J., Mercade M., Vera J., Bueno R., Roig M., Duran-Sindreu F. - Evaluation of the Raypex 5 and the Mini Apex Locator: an in vivo study - Journal of Endodontics, 2011 Oct; 37(10):1349-52.

[20]. Stober E.K., Duran-Sindreu F., Mercade M., Vera J., Bueno R., Roig M. - An evaluation of root ZX and iPex apex locators: an in vivo study - Journal of Endodontics, 2011 May; 37(5):608-10.

[21]. Tinaz A.C., Maden M., Aydin C., Turkoz E. - The accuracy of three different electronic root canal measuring devices: an in vitro evaluation - Journal of Oral Science, 2002 Jun; 44(2):91-5.

[22]. Topuz O., Uzun O., Tinaz A.C., Sadik B. - Oral Surgery, Oral Medicine, Oral Pathology, Oral Radiology, and Endodontics, 2007 Mar; 103(3):73-6.

[23]. Welk A.R., Baumgartner J.C., Marshall J.G. - An in vivo comparison of two frequency-based electronic apex locators - Journal of Endodontics, 2003 Aug; 29(8):497-500. 